\title{
Telemedicine and Physical Examination: A Cautionary Tale and a Prescription for the Future
}

\author{
Ryan $R$ McCool, MD, FACS ${ }^{1,2^{*}}$ \\ ${ }^{1}$ Department of Veterans Affairs Medical Center, White River Junction, Vermont, USA \\ ${ }^{2}$ Geisel School of Medicine at Dartmouth, USA
}

\author{
Keywords \\ Telemedicine, Physical Examination, Cancer, Missed Diagnosis, Otolaryngology
}

\section{Introduction}

The COVID-19 pandemic accelerated the trend toward the remote workplace in many industries, and medicine is no exception. Telemedicine technology, which historically has been used mostly to serve geographically remote populations, such as areas of Alaska that can only be accessed by boat or plane [1], has been rapidly adopted to reach socially distant populations and decrease the risk of viral transmission [2]. Longstanding regulatory and reimbursement blockades to telemedicine have fallen, advancing and improving access. However, as patients and providers embrace this new format, we must be mindful of the technology's blind spots to avoid unintentional harm. I know, because it happened to me early in my telemedicine career.

Several years ago, I evaluated a man in his 60's, who was referred to otolaryngology for a bump on his lip. The patient lived 90 minutes from the hospital and did not have a car, so he relied on the willingness of neighbors to bring him to medical appointments. In order to reduce his travel burden, I was seeing him using telemedicine technology. A trained technician at a satellite clinic near his home examined his ears, nose, and mouth using specialized endoscopes. I interviewed the patient and interpreted the exam from my office at the main hospital in real-time using a dedicated, secure internet connection. That day, my patient described "a bump" he noticed inside his lip about 4 months prior. He said it had not changed in size and was not tender. My exam notes from that day document "pixilation of the video feed," suggesting imperfect internet connection. Nevertheless, I was able to appreciate "incomplete dentition" and "a well-demarcated $4 \mathrm{~mm}$, pale, exophytic lesion on the labial mucosa of the upper lip." Based on his history and telemedicine examination, my differential for his lip lesion included squamous papilloma or traumatic fibroma. Given the small size and benign appearance, I scheduled excisional biopsy for a date 3 months later. This was the soonest he could confirm a ride on a day that I was in clinic.
When my patient presented to the office for the procedure, several things were immediately apparent that I had not been able to appreciate during the telemedicine visit. His complexion was gray, and he smelled strongly of cigarette smoke. Dirty clothes hung loosely from his cache tic frame. I could see now in person that his dentition was not just "incomplete" but in severe decay. Based on these observed risk factors for a head and neck cancer, I repeated his head and neck examination in person. In addition to recognizing the small lip lesion that prompted his consultation, I was dismayed to discover a large, firm, flat tumor that had replaced the normal mucosa of the anterior floor of mouth. The tumor's color matched the adjacent normal mucosa, but unlike the normally smooth, soft mucosa, it was firm with an irregular surface. The tumor was fixed to the nearby mandible but since it did not invade the tongue, his speech and swallow had not yet been impacted. Further workup revealed pronounced erosion of the mandible associated with this T4N1M0 oral cavity squamous cell cancer. His surgical reconstruction after resection of the tumor and bilateral neck dissections required a fibula free flap to replace the resected mandible, followed by radiation therapy.

For this patient, telemedicine enabled accurate diagnosis of his benign lip lesion (squamous papilloma) but arguably delayed care for his life-threatening cancer only a few centimeters away. The cancer, clearly concerning in-person, was not

*Corresponding author: Dr. Ryan R McCool, MD, FACS, Department of Veterans Affairs Medical Center, 215 North Main Street, White River Junction, VT 05009, USA

Accepted: March 13, 2021

Published online: March 15, 2021

Citation: McCool RR (2021) Telemedicine and Physical Examination: A Cautionary Tale and a Prescription for the Future. J Head Neck Surg 3(1):141-142 
evident on telemedicine for several reasons. Video technology then and now cannot convey the full visual and sensory experience of an in-person interaction. One cannot passively recognize a patient's odor or details outside the video frame, such as overall body habitués and carriage. Basic tools like stethoscopes and tongue depressors are not widely available for home telemedicine visits. Here, I had the tools and a trained examiner, and yet the video quality did not allow appreciation of the altered mucosal surface. Most importantly in this case, the loss of palpation severely limited assessment of soft tissues. One of the most important physical signs of cancer, in duration, does not convey at all via telemedicine.

Suboptimal video quality camouflaged my patient's cancer, which was easily recognizable in person. Today, many medical visits occur on video platforms like Zoom, Face Time, or Skype. However, important details can be lost by pixilation or by software designed to intentionally blur surface skin imperfections and optimize coloring. Although some have recently described ways that many elements of a physical examination can be accomplished via telemedicine [3], others have found poor concordance between patient self-exam using consumer devices and office-based physical exam for problems as common as sore throat [4].

To be sure, the expansion of telemedicine eligibility and reimbursement by the Centers for Medicare and Medicaid Services in March of 2020 was an appropriate and necessary response to the health care crisis we are facing. These expansions are arguably overdue and justified to ensure continued access to care. Telemedicine is more convenient and less expensive than office evaluation, and studies consistently demonstrate high patient and provider satisfaction with this format [5]. As a healthcare provider and as a patient, I personally prefer telemedicine for medical appointments that do not require a physical examination.

But stories like this provide an opportunity to redirect our collective focus on patient safety and our oath to do no harm. As we transition out of the emergency response phase of the pandemic, durable telemedicine policy should include minimum standards for video quality when physical examination is performed. Medical societies should define telemedicine use case scenarios for specific disease states within their specialties. Providers should maintain a low threshold for insisting upon office examination if diagnosis cannot be comfortably rendered based on history and review of ancillary lab data. Insurers should not be allowed to deny coverage as duplication of services for the visits that result from telemedicine care that has concerning findings requiring in person follow up. Lastly, providers should create a forum like morbidity and mortality conferences to safely share telemedicine near misses and errors, learn from each other, and improve patient safety.

COVID-19 catalyzed a rapid expansion telemedicine services, and we should expect broader incorporation of video technology into the practice of medicine going forward. Telemedicine offers many advantages over the traditional office visit, and it enables healthcare delivery while reducing the risk of transmission from in-person evaluation. But as we celebrate the conveniences of widespread adoption of telemedicine, let us remain mindful of its important limitations and build appropriate guardrails to protect our patients.

\section{Conflicts of Interest}

I declare that I have no commercial financial conflict of interest.

\section{Disclaimers}

The views expressed do not necessarily represent the views of the Department of Veterans Affairs or the United States Government.

\section{Acknowledgement}

Dr. Louise Davies contributed conceptual input and editing.

\section{Sources of Support}

The Department of Veterans Affairs.

\section{References}

1. Kokesh J, Ferguson AS, Patricoski C, et al. (2009) Traveling an audiologist to provide otolaryngology care using store-and-forward telemedicine. Telemed J E Health 15: 758-763.

2. Mann DM, Chen J, Chunara R, et al. (2020) COVID-19 transforms health care through telemedicine: Evidence from the field. J Am Med Inform Assoc 27: 1132-1135.

3. Benziger CP, Huffman MD, Sweis RN, et al. (2020) The telehealth ten: A guide for a patient-assisted virtual physical examination. Am J Med 134: 48-51.

4. Akhtar M, Van Heukelom PG, Ahmed A, et al. (2018) Telemedicine physical examination utilizing a consumer device demonstrates poor concordance with in-person physical examination in emergency department patients with sore throat: A prospective blinded study. Telemed J E Health 24: 790-796.

5. Kruse CS, Krowski N, Rodriguez B, et al. (2017) Tele health and patient satisfaction: A systematic review and narrative analysis. BMJ Open 7: e016242.

DOI: $10.36959 / 605 / 557$

Copyright: (C) $2021 \mathrm{McCool}$ RR. This is an open-access article distributed under the terms of the Creative Commons Attribution License, which permits unrestricted use, distribution, and reproduction in any medium, provided the original author and source are credited. 